\title{
Avaliação da resiliência do cuidador de idosos com Alzheimer
}

\section{Reilienceedution of caregivers of eddaly peoplewithAtheiner}

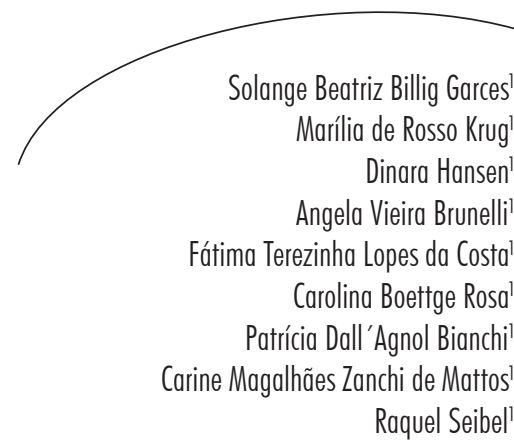

Resumo

Esta pesquisa observacional do tipo estudo de caso descritivo teve o objetivo de avaliar a resiliência de cuidadores de idosos demenciados com Alzheimer. Como instrumento, utilizou-se questionário para traçar o perfil do cuidador; protocolo bipolar para avaliar o estado subjetivo de fadiga; o SRQ - Self Report Questionnaire para avaliação do estresse; o inventário de Zarit para a sobrecarga e protocolo para a resiliência dos seis cuidadores que participaram do estudo. As variáveis quantitativas foram descritas em função de sua média e variância. Para as associações, utilizaram-se Correlação de Pearson e o Teste do Chi-Quadrado. O nível de significância adotado foi de $\mathrm{p} \leq 0,05$. Os resultados indicaram que $83,3 \%$ dos cuidadores são femininos e 16,7\%, masculinos; sendo quatro $(66,7 \%)$ filhos e dois $(33,3 \%)$ cônjuges. Observou-se que o estado de saúde de quatro cuidadores piorou, passando de um estado regular para ruim, em um período de cinco anos. Um (16,7\%) cuidador dedica 20 horas do seu tempo ao cuidado e, para os demais $(83,3 \%)$, a exigência é de 24 horas. Dos seis cuidadores, quatro demonstram probabilidade de desenvolver transtornos psiquiátricos e dois, não. E ainda, 66,7\% dos cuidadores não apresentam sobrecarga, enquanto 33,3\% acham-se com sobrecarga intensa. Embora alguns se mostrem fatigados e sobrecarregados, a maioria (83,3\%) demonstra alta disposição à resiliência. Houve associação significativa entre resiliência e idade $(\mathrm{p} \leq 0,050)$, a qual expressa quanto maior a idade do cuidador maior a resiliência.

\section{Abstract}

This observational study of the descriptive case study type aimed to evaluate the resilience of caregivers of demented elderly people with Alzheimer. A questionnaire was used to trace the profile of the caregivers; bipolar protocol to evaluate the fatigue subjective state; the SRQ - Self Report Questionnaire to evaluate the stress; the Zarit inventory to evaluate overload and protocol to evaluate resilience of the six caregivers
Palavras-chave: Cuidadores. Demência. Fadiga. Resiliência Psicológica.

Grupo Interdisciplinar de Estudos do Envelhecimento Humano. Universidade de Cruz Alta. Cruz Alta, RS, Brasil.

Pesquisa financiada pelo Programa Institucional de Bolsas de Iniciação Científica (PIBIC), da Universidade de Cruz Alta. 
who participated in this study. Quantitative variables were described as a function of their average and variance. For associations, the Pearson Correlation and the ChiSquare test were used. The significance level adopted was $\mathrm{p}<0.05$. Results indicated that $83.3 \%$ of caregivers were females and $16.7 \%$ males, four of which $(66.7 \%)$ sons and two $(33.3 \%)$, consorts. It was observed that the health status of four caregivers got worse, going from a regular to a bad state in a five-year period. One $(16.7 \%)$ caregiver dedicated 20 hours of his time for caring, and for the others (83.3\%) the requirement was of 24 hours. Two did not show any possibility of developing disturbances, while four showed possibilities of developing mental disturbances. $66.7 \%$ of the caregivers did not show overload, while $33.3 \%$ were under intense overload. Even though some demonstrated fatigue and overload, the majority (83.3\%) presented a high disposition for resilience. There was significant association between resilience and age $(p<0.05)$, and this expressed that the older the caregiver the greater the resilience.
Keywords: Caregivers.

Dementia. Fatigue.

Psychological Resilience.

\section{INTRODUÇÃO}

O envelhecimento populacional é uma realidade amplamente conhecida nos países desenvolvidos e se encontra como demanda latente nos países em desenvolvimento. Segundo o Instituto Brasileiro de Geografia e Estatística, ${ }^{1}$ a expectativa de vida no Brasil aumentou cerca de três anos em uma década, passando a ser de 73,1 anos.

É essencial referendar que concorrem para a autonomia nos idosos suas condições de saúde, com destaque para a manutenção da capacidade física e cognitiva. Os grandes causadores da perda de autonomia são as doenças crônicodegenerativas, como hipertensão, diabetes, osteoporose, AVC (acidente vascular cerebral), neoplasia, entre outras.

Dentre as doenças crônico-degenerativas, uma das preocupações trazida pelo envelhecimento é a demência, ${ }^{2}$ especialmente a doença de Alzheimer, prevalente em $10 \%$ da população com mais de 65 anos, sendo que aos 85 anos ou mais esta taxa sobe para 47,2\%. Trata-se de doença neurológica progressiva e irreversível, caracterizada por perdas graduais da função cognitiva e distúrbios do comportamento e do afeto, não sendo facilmente diagnosticada.

Dessa forma, quando o idoso apresenta alguma doença que o deixa dependente, cabe à família apoiá-lo e assumir seu cuidado. $\mathrm{O}$ envelhecimento com dependência resulta em limitações físicas ou cognitivas, e isto leva a uma dependência parcial ou total das atividades da vida diária, gerando demanda de cuidados voltados para as necessidades específicas de cada idoso. Cuidar de idosos significa um processo coletivo e individual de compreensão e aceitação do envelhecimento como algo inerente ao processo existencial. $^{3}$

O cuidador em potencial do idoso dependente são os cônjuges, preferencialmente do sexo feminino, com proximidade física e afetiva. Entretanto, assumem geralmente esta função de forma inesperada e sem qualquer preparação. ${ }^{3}$ Conforme a enfermidade, esta função pode perdurar por muitos anos, o que a torna uma jornada incessante, repetitiva e solitária, bastante desgastante, principalmente quando o cuidador também já é idoso.

Independentemente de o cuidador ser alguém da família ou não, com o passar do tempo a sobrecarga de trabalho lhe trará consequências como fadiga, estresse e depressão, assim este também necessitará de cuidados. Grupos de apoio e psicoterapia se tornam importantes ferramentas para enfrentar as angústias e incertezas do cuidador. ${ }^{3}$

Para enfrentar esses fatores traumatizantes e estressores, entra em cena uma competência que muitos cuidadores apresentam, a chamada resiliência: 
uma capacidade que [...] implica adaptação em face do perigo, a capacidade de sair vencedor de uma prova que poderia ter sido traumática, com uma força renovada. Uma polarização exclusiva nos aspectos vulneráveis dos individuos e nos fatores de risco do seu contexto de vida pode levar a que se negligencie os contributos do estado dos sujeitos que demonstram procedimentos adaptativos e de recursos psiquicos que os preservam dos aspectos psicotóxicos do seu ambiente. ${ }^{4}$

Também se pondera ainda que o núcleo central da resiliência é o fato de trabalhar os potenciais e modos dos sujeitos em face do perigo. ${ }^{4}$ Sabe-se que ser um cuidador domiciliar de idosos dependentes, principalmente os que envolvem doenças demenciais, como o caso do mal de Alzheimer, não é um trabalho de fácil aceitação, o que pode torná-los vulneráveis a sintomas de fadiga, estresse e depressão, mesmo sendo bastante resiliente. Neste sentido, avaliar sintomas de fadiga, estresse e depressão se torna relevante, como forma de ajudar os cuidadores a vencer essas dificuldades e aliviar seu sofrimento.

Considerando que muitas vezes o cuidador é também uma pessoa idosa, torna-se importante direcionar um olhardiferenciado a esta população, no sentido de identificar que o cuidador também merece atenção e que necessário se faz estar bem para conseguir cuidar do outro.

Desta forma, nesta pesquisa buscou-se avaliar os níveis de estresse e fadiga, indícios de depressão e sua consequente capacidade de resiliência mediantes as adversidades cujas atribuições como cuidador de idoso com o mal de Alzheimer lhes impõem.

\section{METODOLOGIA}

Conduziu-se o estudo em um grupo de seis cuidadores de idosos com Alzheimer que frequentam o projeto de extensão da Universidade de Cruz Alta - UNICRUZ, intitulado Estratégias de Diagnóstico e Reabilitação Social de Idosos com Alzheimer e Apoio Psicossocial ao Cuidador, no período de maio a novembro de 2010. Tratase, portanto, de pesquisa observacional do tipo estudo de caso descritivo (série de casos).
Como instrumentos de pesquisa, utilizou-se um questionário para traçar o perfil do cuidador familiar, estabelecendo as atividades desenvolvidas com o idoso dependente, ${ }^{5}$ o protocolo bipolar ${ }^{6}$ validado em 1998 para avaliar o estado subjetivo de fadiga; o Self Report Questionnaire (SRQ) elaborado por Harding et al. em 1980 e validado no Brasil por Mari \& Willians, para avaliação do estresse; o inventário de Zarit ${ }^{8}$ para a sobrecarga dos cuidadores e um protocolo para a capacidade de resiliência dos cuidadores de idosos dependentes, desenvolvido por Wagnild e Young e adaptado e validado por Pesce et al. ${ }^{9}$

Realizou-se análise estatística dos dados através do programa SPSS versão 17.0. Descreveram-se as variáveis quantitativas em função de sua média e variância. A verificação de possíveis associações entre as variáveis quantitativas foi estimada através do coeficiente de Correlação de Pearson. Também se calculou a associação entre as variáveis qualitativas nominais através do Teste do $\mathrm{X}^{2}$ (Chiquadrado). O nível de significância adotado foi de $\mathrm{p}$ $\leq 0,05$. E ainda, foram analisadas estatisticamente pelo Teste $\mathrm{t}$ de Student as diferenças eventuais entre médias dos sujeitos agrupados segundo as variáveis categóricas.

As questões éticas permearam todo o estudo, seguindo os preceitos da Resolução n. 196/96 do Conselho Nacional de Saúde, que trata das Diretrizes e Normas Regulamentadoras de Pesquisa com Seres Humanos. Como este projeto de pesquisa integrou o de extensão Estratégias de Diagnóstico e Reabilitação Social de Idosos com Alžheimer e Apoio Psicossocial ao Cuidador, foi encaminhado ao CEP da UNICRUZ para sua apreciação ética e recebeu aprovação sob registro do CAAE $n^{\circ}$ 0038.0.417.000-10.

\section{RESULTADOS E DISCUSSÕES}

\section{Perfil e dados socioeconômicos dos cuidadores}

Realizou-se a pesquisa com um grupo de seis cuidadores de idosos com provável ou possível diagnóstico de Alzheimer. Em relação ao gênero, predominou o feminino, cinco e um masculino e, destes, quatro filhos e dois cônjuges. Esses 
resultados demonstram que todos os cuidadores possuem relação de parentesco de primeiro grau com o idoso demente, recaindo o cuidado mais especificamente para as filhas mulheres. $\mathrm{O}$ cuidador, cônjuge do sexo masculino, dispõe de apoio de uma cuidadora sem grau de parentesco, pois o mesmo apresenta problemas de saúde que impossibilitam a realização de todos os cuidados.

A responsabilidade do cuidado de pacientes idosos recai sobre a mulher, seja esposa, filha ou irmã. ${ }^{10}$ Assim, $80 \%$ dos cuidadores de idosos com síndrome demencial são familiares, e os membros da família representam 90\% de cuidadores, ${ }^{11}$ conforme estudo sobre cuidadores de pacientes com Alzheimer. ${ }^{11,12}$ Em outra pesquisa realizada, também se constatou que $89,7 \%$ dos cuidadores de idosos com Alzheimer do sexo feminino estão com idade média entre 48 e 58 anos, sendo que destes a maior parte eram filhos ou cônjuges. ${ }^{2}$

Nesta linha de percepção, em estudos com cuidadores informais, verificou-se que a maioria reside com o idoso doente, mulheres, filhas ou esposas, casados, com idade média entre 55 e 65 anos. $^{12,13}$ As pesquisas em grande parte apresentam-se concordantes quanto ao sexo e ao grau de parentesco dos cuidadores. ${ }^{12,14-16} \mathrm{Em}$ outro estudo italiano, $80 \%$ a $90 \%$ dos cuidadores são familiares do idoso doente, sendo $70 \%$ do sexo feminino. ${ }^{17}$

O fato de um membro da família desencadear um processo de dependência altera a dinâmica familiar. Em geral, são as mulheres que assumem o cuidado, e esse papel é visto como natural, pois se infere socialmente no papel de mãe e de filha. Outro fato importante centra-se na faixa etária dos cuidadores que pertencem à mesma geração dos doentes, fazendo assim com que idosos jovens independentes cuidem de idosos dependentes. ${ }^{18}$ Também esta pesquisa demonstra que para $50 \%$ dos cuidadores, a faixa etária de idade é de mais de 60 anos. A média de idade foi de 59,5 \pm 7,96, com a mínima de 48 e a máxima 69 anos, conforme evidencia a tabela 1.

Tabela 1 - Perfil dos Cuidadores. Cruz Alta, RS, 2010.

\begin{tabular}{llcc}
\hline Variável & Descrição & Frequência & Percentual (\%) \\
\hline Sexo & Masculino & 01 & 16,7 \\
& Feminino & 05 & 83,3 \\
Estado Civil & Casado & 03 & \\
& Solteiro & 01 & 50,0 \\
& Viuvo & 01 & 16,70 \\
& Separado & 01 & 16,70 \\
& & & 16,70 \\
Profissão & Doméstica & 03 & 50,0 \\
& Agropecuarista & 01 & 16,70 \\
& Do lar & 01 & 16,70 \\
& Professora & 01 & 16,70 \\
Faixa Etária & & & \\
& & 01 & 16,7 \\
& 40 a 49 anos & 02 & 33,0 \\
& 50 a 59 anos & 03 & 50,0 \\
\hline
\end{tabular}


Em referência ao estado civil, 50\% são casados e moram junto com os idosos dos quais são cuidadores, sendo que, destes, quatro $(66,7 \%)$ declaram-se proprietários das residências e dois $(33,3 \%)$, residem em casas emprestadas por parentes. A média da renda dos entrevistados é de 3,8 salários mínimos, e apenas um deles apresenta condição financeira maior (agropecuarista). A profissão que cada cuidador exercia antes de se dedicar ao cuidado também se encontra na tabela 1.

Os resultados da pesquisa evidenciaram que, dos cuidadores entrevistados, quatro são filhas e estas trouxeram seus pais dependentes para morar em suas residências. Neste caso, há o predomínio de uma "solidariedade imposta" por pressão de saúde. ${ }^{19}$ Em pesquisa realizada sobre cuidadores de idosos demenciados, houve predominância de mulheres, filhas ou esposas e em corresidência com estes. ${ }^{12}$ Outros estudos igualmente identificaram o papel da família, e especialmente da mulher, exercendo o papel de cuidador principal..$^{10,20-22}$

A média de filhos de cada cuidador é de $3,16 \pm 1,16$, sendo que o máximo é cinco e o mínimo dois, e a convivência entre os familiares é semanal, geralmente no final de semana.

Em relação à escolaridade, cinco (83,3\%) apresentam ensino fundamental incompleto $\mathrm{e}$ uma $(16,7 \%)$ das cuidadoras apresenta o ensino médio incompleto. Esta era professora leiga da zona rural antes de cuidar da mãe com Alzheimer.

O cuidador familiar se revela $\mathrm{o}$ ator social principal no atendimento de pacientes dependentes, mas cuidar de um indivíduo idoso durante muitas horas não pode ser tarefa de uma mulher sozinha, sem apoio e suporte para atender a suas necessidades, ${ }^{23}$ por isso necessário se faz conhecer as condições de saúde das cinco cuidadoras e um cuidador, sujeitos desta pesquisa.

\section{Condições de saúde do cuidador}

Cuidar de idosos dependentes, especialmente aqueles que apresentam demência, não é tarefa fácil e como geralmente recai sobre os membros mais próximos da família, acabam estes sendo, de certa forma, sobrecarregados em relação aos demais. Esta sobrecarga acumulada prejudica a saúde, gerando mal-estar e piora do seu estado físico e emocional.

A tabela 2 apresenta as percepções dos cuidadores pesquisados em relação ao seu estado de saúde atual, comparando-o ao de cinco anos anteriores. Observa-se que o estado de saúde de quatro cuidadores piorou, passando de regular para ruim.

Tabela 2 - Percepção do estado de saúde dos cuidadores. Cruz Alta, RS, 2010.

\begin{tabular}{llcc}
\hline Variável & Descrição & Frequência & Percentual (\%) \\
\hline Estado de saúde & Regular & 04 & 66,7 \\
cinco anos atrás & Ruim & 01 & 16,7 \\
& Péssima & 01 & 16,7 \\
& & & \\
Estado de saúde & ruim & 05 & 83,3 \\
atual & péssima & 01 & 16,7 \\
\hline
\end{tabular}


Infelizmente, as más condições de saúde do cuidador constituem fortes preditores de institucionalização do idoso demenciado. ${ }^{12}$ Quando isto acontece, há geralmente um agravamento da doença no idoso demenciado.
Para conhecer as condições de saúde dos cuidadores, questionou-se sobre internações referentes ao último ano e consultas médicas nas últimas duas semanas. Conforme resultados apresentados na tabela 3, observa-se que entre os cuidadores nenhum se internou no último ano, porém em $50 \%$ deles houve duas ocorrências em quatro anos.

Tabela 3 - Variáveis relacionadas a saúde do cuidador. Cruz Alta, RS, 2010.

\begin{tabular}{lccc}
\hline \multicolumn{1}{c}{ Variável } & Descrição & Frequência & Percentual (\%) \\
\hline Convênio de & Sim & 02 & 33,3 \\
saúde & Não & 04 & 66,7 \\
& Sim & 00 & 0,00 \\
Esteve acamado & Não & 06 & 100,0 \\
Procurou Médico & Procurou e foi atendido & 03 & 50,0 \\
& Não procurou & 03 & 50,0 \\
Número de & Nenhuma & 01 & 16,7 \\
consultas médicas & 1 vez & 01 & 16,7 \\
& 2 a 3 vezes & 01 & 16,7 \\
& 4 a 6 vezes & 01 & 16,7 \\
Número de internações & Mais de 6 vezes & 02 & 33,3 \\
hospitalares no último ano & Nenhuma & 06 & 100,0 \\
& 1 vez & 00 & 0,00 \\
Teve internação hospitalar & 2 vezes & 00 & 0,00 \\
nos últimos quatro anos & Mais de 2 vezes & 00 & 0,00 \\
\hline
\end{tabular}

Perfil de patologias apresentadas pelo cuidador e uso de medicações

Para identificar a presença de doenças (morbidades), perguntou-se aos cuidadores sobre doenças que já apresentaram ou apresentam, diagnosticadas por médico, conforme tabela 4.

Observa-se que as morbidades que aparecem com maior frequência são doenças crônicas não-transmissíveis identificadas com a idade do cuidador, como a artrite e reumatismo, osteoporose, trombose e varizes. Já a úlcera, a gastrite e a depressão relacionam-se à condição de estresse e fadiga que geralmente acomete os cuidadores. Uma das cuidadoras apresenta outra doença que a torna também depressiva, necessitando de acompanhamento psicológico.

Assim, as tarefas desenvolvidas pelo cuidador, ao longo do tempo e associadas a diversos fatores, acabam constituindo eventos estressores significativos. $^{2}$ Isso os tornam também pacientes e os submete a tratamentos com uso de medicamentos diários, conforme exposto na tabela 5, segundo aqual 50\% destes fazem uso de dois a quatro medicamentos diferentes. 
Tabela 4 - Morbidades declaradas pelos cuidadores. Cruz Alta, RS, 2010.

\begin{tabular}{lcc}
\hline \multicolumn{1}{c}{ Patologia } & Frequência & Percentual (\%) \\
\hline Artrite/Doenças Reumáticas & 4 & 66,7 \\
Depressão & 4 & 66,7 \\
Ulcera/Gastrite & 4 & 66,7 \\
Trombose/Varizes & 4 & 66,7 \\
Osteoporose & 3 & 50,0 \\
Alergia & 2 & 33,3 \\
Fraturas & 2 & 33,3 \\
Insuficiência Cardíaca & 2 & 33,3 \\
Diabetes & 1 & 16,7 \\
Dislipidemia & 1 & 16,7 \\
Angina & 1 & 16,7 \\
Infarto agudo do miocárdio & 1 & 16,7 \\
Constipação & 1 & 16,7 \\
Gripe no último ano & 1 & 16,7 \\
Tireoidismo & 1 & 16,7 \\
Outra & 1 & 16,7 \\
\hline
\end{tabular}

Tabela 5 - Uso de medicamentos pelos cuidadores. Cruz Alta, RS, 2010.

\begin{tabular}{cccc}
\hline Variável & Descrição & Frequência & Percentual (\%) \\
\hline Toma remédios & Sim & 4 & 66,7 \\
diariamente & Não & 2 & 33,3 \\
& & & \\
Nenhum & 2 & 33,3 \\
Número de & Até 02 & 0 & 0,00 \\
medicamentos ingere & 2 a 4 & 3 & 50,0 \\
por dia & 4 a 6 & 0 & 0,00 \\
& Mais de 6 & 1 & 16,7 \\
\hline
\end{tabular}

Constatou-se, portanto, serem sintomas físicos mais comuns em cuidadores: a hipertensão, desordens digestivas, doenças respiratórias e propensão a infecções. ${ }^{24}$ Assim como os sintomas físicos citados no estudo destes autores, também prevaleceram nesta pesquisa as desordens digestivas. 
Perfil e rotina de cuidado

À medida que a doença progride, as regressões se mostram mais evidentes e diminui nos pacientes a capacidade de realizar as atividades básicas e instrumentais da vida diária. Salienta-se que a rotina de tarefas e cuidados compreende a preparação e a administração da alimentação, o banho, o vestir-se, a administração da própria vida, do dinheiro, das compras, dos medicamentos, da limpeza da casa, em alguns casos, a troca de fraldas e auxílio para ir ao banheiro, conforme mostra a tabela 6 .

Tabela 6 - Tarefas que a rotina de cuidados exige. Cruz Alta, RS, 2010.

\begin{tabular}{lcccc}
\hline \multirow{2}{*}{ Variável } & \multicolumn{2}{c}{$\operatorname{Sim}$} & \multicolumn{2}{c}{ Não } \\
\cline { 2 - 5 } & $\mathrm{n}$ & $\%$ & $\mathrm{n}$ & $\%$ \\
\hline - Alimentação & 6 & 100,0 & 0 & 00,0 \\
- Banho & 6 & 100,0 & 0 & 00,0 \\
- Vestir-se & 6 & 100,0 & 0 & 00,0 \\
- Trocar fraldas & 3 & 50,0 & 3 & 50,0 \\
- Ajuda para ir o banheiro & 4 & 66,7 & 2 & 33,3 \\
- Administrar a própria vida & 6 & 100.0 & 0 & 00,0 \\
- Locomover/Deslocar & 5 & 83,3 & 1 & 16,7 \\
- Cuidar das finanças & 6 & 100.0 & 0 & 00,0 \\
- Fazer compras & 6 & 100.0 & 0 & 00,0 \\
- Administrar medicamentos & 6 & 100,0 & 0 & 00,0 \\
- Limpeza da Casa & 6 & 100,0 & 0 & 00,0 \\
- Preparar alimento & 6 & 100,0 & 0 & 00,0 \\
\hline
\end{tabular}

Essas tarefas fazem com que o cuidado seja contínuo e rotineiro, exigindo cada vez mais dedicação e paciência e, em decorrência, o cuidador passa a viver a vida do idoso. Em estudo sobre as perspectivas do cuidador de idoso com Alzheimer, observou-se que na maioria das vezes, a linha tênue de divisão entre a vida do paciente e a do cuidador desaparece, pois o cuidador passa a experimentar a vida de seu familiar doente intensamente, a fim de que nada lhe falte. ${ }^{25}$

Dentre os idosos atendidos pelos cuidadores pesquisados, um se encontra na fase final da doença de Alzheimer, uma na fase inicial, e os demais, na fase secundária. Dessa forma, dos seis cuidadores, um $(16,7 \%)$ dedica 20 horas do seu tempo ao cuidado e para os demais $(83,3 \%)$ a exigência é de 24 horas.
Em estudo com cuidadores de idosos com Alzheimer, constatou-se que além das tarefas no domićlio e dos problemas familiares, essas mulheres cuidam na maioria das vezes, integral e diariamente do idoso com Alzheimer, evidenciando também o gênero feminino como condicionante ao cuidado e dedicação dioturnamente. ${ }^{26}$

\section{Consequências do cuidado}

Outros aspectos que se destacam são os comportamentos mais frequentes dos idosos que apresentam Alzheimer. Muitos cuidadores relataram que os idosos se tornam agressivos verbalmente, reclamando da situação, não 
gostam de tomar banho, tornam-se teimosos, repetem histórias e principalmente esquecem de tomar os remédios, não sabem onde guardam as coisas, além de inventarem histórias e fantasias.
Esses comportamentos assustam os cuidadores, sobretudo em situações em que faltam informações mais detalhadas sobre a doença e sua evolução, conforme descrito na tabela 7 .

Tabela 7 - Comportamentos mais frequentes do idoso. Cruz Alta, RS, 2010.

\begin{tabular}{lcccc}
\hline \multicolumn{1}{c}{ Variável } & \multicolumn{2}{c}{ Sim } & \multicolumn{2}{c}{ Não } \\
\cline { 2 - 5 } & $\mathrm{n}$ & $\%$ & $\mathrm{n}$ & $\%$ \\
\hline Reclama da situação & 6 & 100,0 & 0 & 0,00 \\
Junta coisas inúteis & 3 & 50,0 & 3 & 50,0 \\
Apresenta alterações de sono & 3 & 50,0 & 3 & 50,0 \\
Agressivo verbalmente & 3 & 50,0 & 3 & 50,0 \\
Come coisas que não deve & 1 & 16,7 & 5 & 83,3 \\
Não gosta de banho & 3 & 50,0 & 3 & 50,0 \\
Não se importa com limpeza & 3 & 50,0 & 3 & 50,0 \\
Repete histórias & 4 & 66,7 & 2 & 33,3 \\
Suja roupas com urina e fezes & 4 & 66,7 & 2 & 33,3 \\
Necessita troca de fraldas urinárias & 3 & 50,0 & 3 & 50,0 \\
Esquece rotina dos remédios & 6 & 100,0 & 0 & 0,0 \\
Não consegue achar coisas & 4 & 66,7 & 2 & 33,3 \\
Não quer sair do quarto & 2 & 33,3 & 4 & 67,7 \\
Vive chorando & 1 & 16,7 & 5 & 83,3 \\
Comportamento agressivo & 1 & 16,7 & 5 & 83,3 \\
Teimosia & 5 & 83,3 & 01 & 16,7 \\
\hline
\end{tabular}

Estudos sobre cuidadores relatam que as modificações percebidas no comportamento dos idosos foram a agressividade oral e física, persistência, teimosia, choro, gritos, desorientação no espaço (se perde), esquecimento, não usar o telefone corretamente e alucinações. ${ }^{26}$ Em algumas situações em que os cuidadores demoram, acrescentam-se angústias, depressão e conflitos que conduzem à fadiga e ao estresse. A partir do momento que os cuidadores tomam conhecimento sobre a evolução da doença e suas características, a convivência é facilitada pela preparação e organização das mudanças necessárias para o cuidado. ${ }^{25}$
O estresse é utilizado como sinônimo de sobrecarga do cuidador, sendo também chamado de fadiga e impacto. Refere-se à presença de problemas, dificuldades ou eventos adversos que afetam significativamente a vida de pessoas que são responsáveis pelo paciente. ${ }^{27}$ O estresse é uma das queixas mais frequentes de cuidadores relatados em vários estudos ${ }^{26-28}$ e também nesta pesquisa, contribuindo para isso uma série de atividades importantes para o bem-estar das pessoas, pois muito cuidadores abdicam de amigos, férias, lazer e ocupação profissional, conforme dados apresentados na tabela 8 . 
Tabela 8 - Comportamentos mais frequentes do-cuidador. Cruz Alta, RS, 2010.

\begin{tabular}{lcccc}
\hline \multicolumn{1}{c}{ Variável } & \multicolumn{2}{c}{ Sim } & \multicolumn{3}{c}{ Não } \\
\cline { 2 - 5 } & $\mathrm{n}$ & $\%$ & $\mathrm{n}$ & $\%$ \\
\hline Redução do lazer & 6 & 100,0 & 0 & 0,00 \\
Sente-se sempre cansado(a) & 3 & 50,0 & 3 & 50,0 \\
Sem tempo para os amigos & 5 & 83,3 & 1 & 16,7 \\
Não tem férias & 6 & 100,0 & 0 & 0,00 \\
Sente sua saúde piorada & 5 & 83,3 & 1 & 16,7 \\
Não pode trabalhar fora & 5 & 83,3 & 1 & 16,7 \\
Sem tempo para cuidar de si & 4 & 66,7 & 2 & 33,3 \\
Sente-se deprimida & 3 & 50,0 & 3 & 50,0 \\
Aumento dos problemas econômicos & 5 & 83,3 & 1 & 16,7 \\
Conflitos familiares & 2 & 33,3 & 4 & 66,7 \\
Teve que parar de trabalhar fora & 5 & 83,3 & 1 & 16,7 \\
Sente-se estressada(o) & 5 & 83,3 & 1 & 16,7 \\
\hline
\end{tabular}

Indicadores de depressão, estado subjetivo de fadiga, transtornos mentais

e sobrecarga do cuidador

Para analisar o estado subjetivo de fadiga do cuidador, aplicou-se o questionário bipolar, que avalia seu estado emocional através do questionamento por meio de palavras identificadoras de comportamentos antagônicos, como cansado e descansado, por exemplo. A estimativa do estado subjetivo de fadiga dos cuidadores é apontada nos gráficos apresentados nas figuras 1 e 2, onde se destacam os estados de cansaço e esgotamento dos cuidadores. 

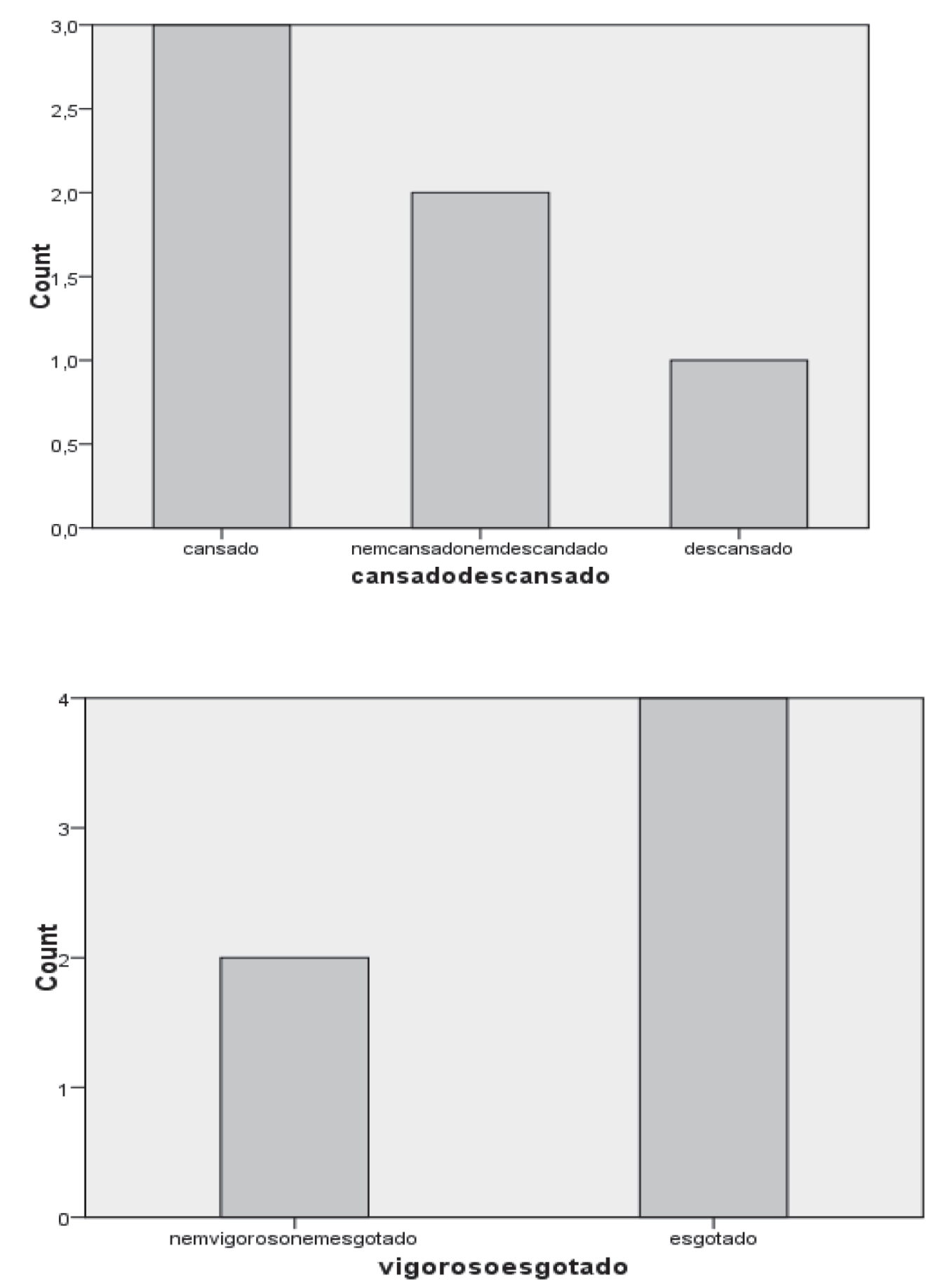

Figuras 1 e 2 - Gráficos referentes ao estado subjetivo de fadiga do cuidador 
Dos resultados apresentados, é interessante destacar que $50 \%$ dos cuidadores encontram-se cansados; no entanto, $50 \%$ deles encontram-se despertos, pois o próprio cuidado assim o exige. Já 66,6\% encontram-se esgotados com a tarefa do cuidado, assim como também se sentem fracos. Entretanto, 33,3\% deles informam se sentirem com energia para o cuidado; 33,3\% consideram-se estimulados; $66,6 \%$ encontramse interessados; $50 \%$, atentos e os outros $50 \%$, distraídos. Estes resultados demonstram que o estado subjetivo de fadiga do cuidador se mostra mais intenso naqueles em que o idoso exige maior cuidado e em que a doença de Alzheimer se encontra mais avançada, e especialmente naqueles sem ninguém com quem dividir as tarefas do cuidado.

Pondera-se que problemas sociais, piora da saúde física e sintomas psiquiátricos principalmente depressão -, são as consequências mais comuns do impacto de cuidar de um dementado. ${ }^{12}$ Assim, para avaliar a presença de transtornos mentais entre os cuidadores, aplicou-se o Self Report Questionnaire (SRQ). Este questionário identifica a probabilidade da presença de transtornos mentais, ou seja, distúrbios psiquiátricos em nível de atenção primária; foi desenvolvido por Harding et al. em 1980 e validado no Brasil por Mari \& Willians. ${ }^{4}$ O instrumento de autorrelato apresenta 20 questões, com alternativas sim (01 ponto) e não (0 ponto), onde o participante responde referente aos sintomas do último mês. Tem como ponto de corte 7 para o sexo feminino e 6 para o masculino. Este ponto de corte permite a obtenção de dois grupos: de um lado, os indivíduos com maior probabilidade de ter um transtorno mental comum, e de outro, um grupo com maior probabilidade de não o ter.

Apresentaram $\geq 7$ respostas positivas 66,6\% dos cuidadores, sendo todas mulheres. Isto significa dizer que nesta pesquisa com os cuidadores de idosos com Alzheimer, dois não apresentam probabilidade de desenvolver transtornos psiquiátricos, enquanto quatro ficaram com pontuação acima do ponto de corte, indicando que se encontram com probabilidade de desenvolverem transtornos mentais.

Divide-se este questionário em quatro dimensões, tendo questões específicas para cada uma. Os percentuais apresentados pelos cuidadores pesquisados, em cada dimensão, encontram-se na tabela 9 . 
Tabela 9 - Frequência de respostas afirmativas do SRQ-20. Cruz Alta, RS, 2010.

\begin{tabular}{lcc}
\hline \multicolumn{1}{c}{ Fatores do SRQ-20 Humor Depressivo } & Frequência & Percentual (\%) \\
\hline $\begin{array}{l}\text { Assusta-se com facilidade } \\
\text { Sente-se nervoso, tenso e preocupado }\end{array}$ & 5 & 83,3 \\
Tem se sentido triste ultimamente & 5 & 83,3 \\
Tem chorado mas do que de costume & 4 & 66,6 \\
$\quad$ & 4 & 66,6 \\
Tem dóticos & & \\
Tem falta de cabeça frequentes & 2 & 44,4 \\
Dorme mal & 0 & 0,00 \\
Tem tremores de mão & 4 & 66,6 \\
Tem má digestão & 1 & 16,6 \\
Tem sensações desagradáveis no estômago & 1 & 16,6 \\
$\quad$ Decréscimo de energia vital & 4 & 66,6 \\
Tem dificuldade de pensar com clareza & & \\
Tem dificuldade para realizar com satisfação suas atividades diárias & 3 & 50,0 \\
Tem dificuldades para tomar decisões & 4 & 66,6 \\
Seu trabalho diário lhe causa sofrimento & 4 & 66,6 \\
Sente-se cansado o tempo todo & 2 & 44,4 \\
Se cansa com facilidade & 2 & 44,4 \\
$\quad$ Pensamentos Depressivos & 4 & 66,6 \\
É incapaz de desempenhar um papel útil em sua vida & & 16,6 \\
Tem perdido o interesse pelas coisas & 1 & 44,4 \\
Você se sente pessoa inútil & 2 & 0,00 \\
Tem tido ideia de acabar com a vida & 0 & 16,6 \\
\hline
\end{tabular}

Destacam-se nas respostas dos cuidadores a maior frequência, para os cuidadores, do humor depressivo-ansioso e decréscimo de energia vital. Pensamento depressivo indicativo de uma das cuidadoras que, além do cuidado, apresenta doença que a deixa depressiva, inclusive com acompanhamento psicológico. Entende-se que esta cuidadora necessita de cuidados para si, não apresentando condições de cuidar de um idoso com Alzheimer. Em pesquisa conduzida por Garrido \& Menezes, o escore médio do SRQ foi de 6,3 enquanto neste estudo foi de $8,8 .^{12}$

Os resultados do Zarit Burden Interview aplicado neste estudo indicaram que 66,7\% dos cuidadores não apresentam sobrecarga, enquanto 33,3\% estão com sobrecarga intensa, conforme figura 3 . 


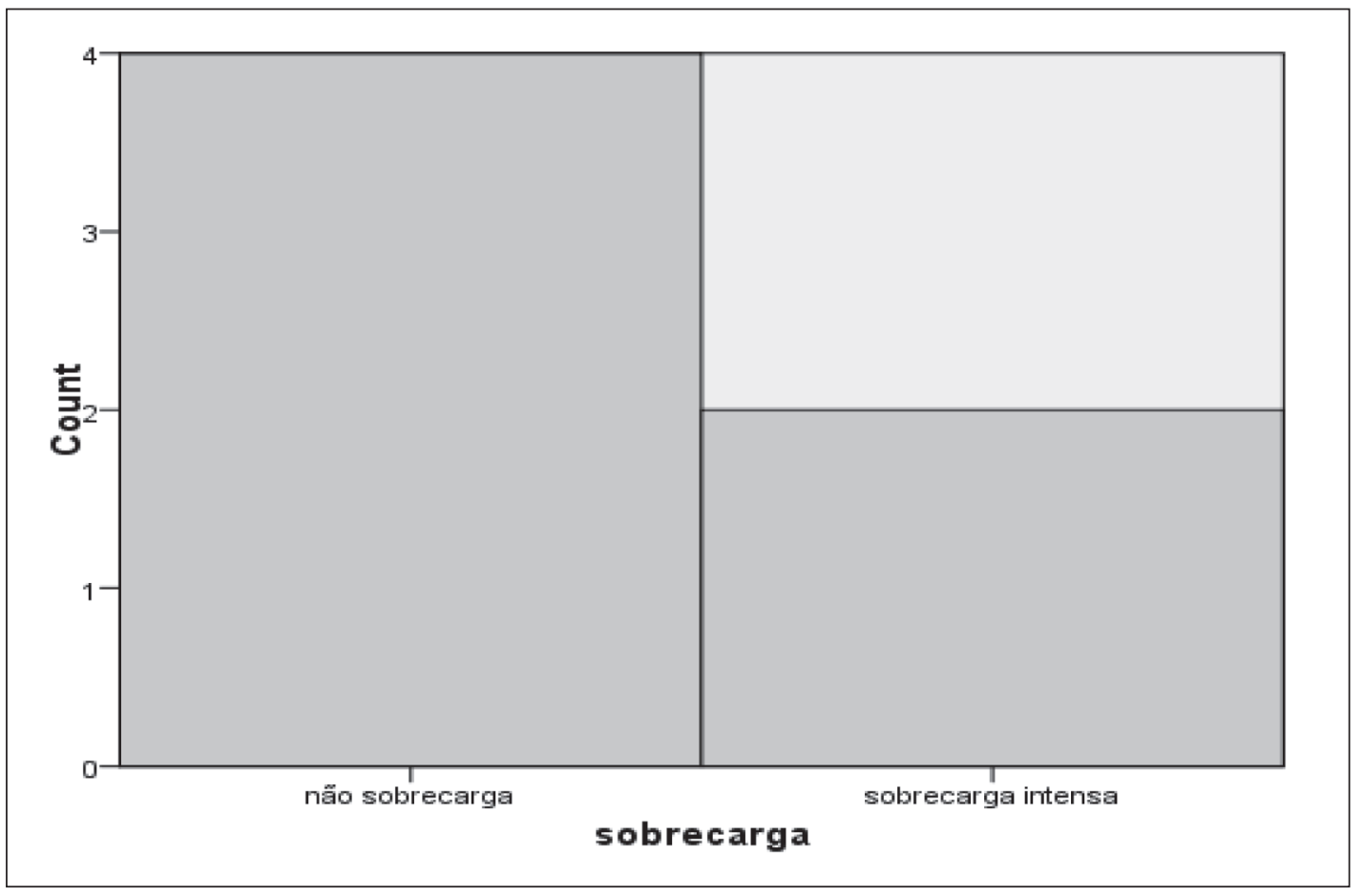

Figura 3 - Gráfico que avalia sobrecarga dos cuidadores através do protocolo Zarit Burden Interview

No estudo realizado por Garrido \& Menezes, ${ }^{12}$ que também utilizou o Zarit Burden Interview, verificou-se que quando questionados sobre a frequência com que, de maneira geral, se sentiram sobrecarregados por cuidar do dementado, os cuidadores dividiram-se entre os extremos da escala: $21(42,8 \%)$ responderam "nunca ou raramente" e 19 (38,7\%) "frequentemente ou sempre". Tais resultados vêm ao encontro dos achados desta pesquisa.

\section{Resiliência do cuidador}

Para tolerar as atividades que a rotina do cuidado de uma pessoa demenciada exige, os cuidadores precisam apresentar atitudes que os façam suportar a sobrecarga de tarefas decorrentes desta com atitudes positivas e se mostrando resilientes em relação à situação. Indaga-se aqui o significado de ser resiliente.

Utiliza-se o conceito de resiliência em diferentes ações sociais, educativas e de saúde, que englobam indivíduos de todas as idades, da infância à velhice. A resiliência liga-se ao desenvolvimento e ao crescimento humano. As condutas de resiliência requerem fatores de resiliência e ações. Estas supõem a presença e a interação dinâmica de fatores, e estes mudam nas diferentes etapas do desenvolvimento. As situações de adversidade não são estáticas, mudam e requerem mudanças nas condutas resilientes. Assim, ser resiliente exige se preparar, viver e aprender com as experiências adversas, como mudança de país, doença ou abandono. ${ }^{29}$

Resiliência seria um resultado da interação entre fatores genéticos e ambientais, o que se configura como algo complexo, visto que estes podem atuar tanto como fatores de proteção para o indivíduo como de risco. Por isso, alguns indivíduos, em determinado momento de sua vida, apresentam-se resilientes e em outros não, pois se as circunstâncias mudam, a resposta da pessoa também pode ser modificada. Dessa forma, qualquer pessoa, em algum momento da sua vida, mostra-se resiliente, uma vez que a resiliência não é um construto universal aplicável a todas as áreas do funcionamento humano, já que se trata de um processo muito dinâmico, multifatorial e multidimensional. ${ }^{30}$ Pode-se, 
portanto, definir resiliência como sendo a capacidade humana para enfrentar, vencer e ser fortalecido ou transformado por experiências de adversidade, e a maioria das definições do conceito de resiliência é uma variação desta. ${ }^{29}$

O papel da resiliência é desenvolver a capacidade humana de enfrentar, vencer e sair fortalecido de situações adversas e transformado. É um processo que excede o simples "superar" essas experiências, já que permite sair fortalecido por elas, o que necessariamente afeta a saúde mental. Assim, embora alguns dos cuidadores do estudo se apresentem fatigados e sobrecarregados, a motivação de continuar e persistir na tarefa do cuidado com certeza é sua capacidade de resistir aos desafios e provações que a vida lhes oferece, pois a maioria $(83,3 \%)$ apresentou alta disposição à resiliência, conforme aponta a tabela 10 .

Tabela 10 - Resiliência. Cruz Alta, RS, 2010.

\begin{tabular}{ccc}
\hline Resiliência & Frequência & Percentual $(\%)$ \\
\hline Baixa & 0 & 0,00 \\
Moderada & 01 & 16,7 \\
Alta & 05 & 83,3 \\
\hline
\end{tabular}

Autores descobriram que as pessoas resilientes demonstraram capacidade de enfrentar adversidades. Advertiram também que a resiliência reduzia a intensidade do estresse e a presença de sinais emocionais negativos, como ansiedade, depressão ou raiva, ao mesmo tempo em que aumentava a curiosidade e a saúde emocional. Portanto, a resiliência é efetiva não apenas para enfrentar adversidades, mas também para promover saúde mental e emocional. ${ }^{31}$

\section{Associação entre resiliência e as demais variáveis}

Para identificar a associação significativa entre resiliência e as demais variáveis, associaram-se os dados sociodemográficos como idade, sexo, escolaridade, estado civil, grau de parentesco, profissão, número de filhos, com quem vive e estado de saúde atual com a resiliência.

Houve associação significativa entre resiliência e idade $(\mathrm{p} \leq 0,050)$, o que expressa que quanto mais idade o cuidador tem, mais resiliente se mostra. Nas demais variáveis não houve associação significativa, embora quando associadas separadamente as questões que compõem o questionário da resiliência e sexo, houve diferença significativa em algumas delas, conforme se observou: "Eu raramente penso sobre os objetivos das coisas" ( $\mathrm{p} \leq$ 0,014); "Em uma emergência, eu sou uma pessoa em quem as pessoas podem contar" ( $\mathrm{p} \leq 0,050)$; “Eu posso geralmente olhar uma situação de diversas maneiras" ( $\mathrm{p} \leq 0,050)$; e "Às vezes me obrigo a fazer coisas querendo ou não $(p \leq 0,050)$ ".

Quando associadas separadamente as alternativas que compõem o questionário da resiliência e idade, houve diferença significativa: "Eu sou amigo de mim mesmo" ( $\leq \leq 0,050)$ e "Eu faço as coisas um dia de cada vez" $(\mathrm{p} \leq 0,050)$. Isto demonstra que a idade torna as pessoas mais resilientes e portanto, mais resistentes às adversidades.

Ainda houve associação significativa na questão "Eu sinto orgulho de ter realizado coisas em minha vida", do questionário da resiliência e o grau de escolaridade dos cuidadores $(\mathrm{p} \leq$ $0,050)$. Este resultado indica que embora nem todos tenham um alto nível de escolaridade, 
consideram que fizeram o máximo que podiam nos diferentes momentos de suas vidas e por isso se orgulham do que são hoje.

Também houve associação significativa quando se relacionou o Estado de Saúde atual e as questões do instrumento da resiliência " $\mathrm{Eu}$ sinto orgulho de ter realizado coisas em minha vida" ( $\mathrm{p} \leq 0,017)$ e "Eu sou determinado" ( $\mathrm{p} \leq$ $0,050)$, indicando que as mulheres se consideram determinadas e orgulhosas no que se refere às condições para o cuidado.

Quando se associou o instrumento Self Report Questionnaire (SRQ), que avalia a probabilidade da presença de transtornos mentais entre os cuidadores e o instrumento da resiliência, não houve associação significativa entre estes, embora quando analisadas algumas questões separadamente, houve associações significativas. As questões relacionadas a autoestima, disciplina e confiança em si mesmo e que dão o suporte para o enfrentamento da fadiga e da sobrecarga foram as que apresentaram os resultados mais significativos.

Em estudo de revisão da literatura sobre o impacto do cuidado, foram encontrados sete artigos $(50,0 \%)$ que registraram relação positiva entre transtornos de comportamento do paciente e desenvolvimento de sintomas depressivos no cuidador, e sete estudos $(50,0 \%)$ que sugerem que a sobrecarga se associa ao comprometimento das atividades da vida diária do paciente. ${ }^{31}$

No presente estudo, quando se dicotomizaram as variáveis para associar o Zarit Burden Interview e o SRQ, não houve associação significativa (Quiquadrado $=\mathrm{p} \leq 0,221$ e o teste de Fischer $=0,467$ ). No entanto, houve associação significativa entre o SRQ e moradia ( $\mathrm{p} \leq 0,014)$, e entre o SRQ e doenças $(0,014)$, mas não houve associação significativa entre o SRQ e o sexo dos cuidadores $(\mathrm{p} \leq 0,121)$. Também não houve associação significativa entre a resiliência e o SRQ $(\mathrm{p}<0,439)$, nem entre o Zarit Burden Interview e a resiliência $(0,439)$.

Em relação ao Zarit Burden Interview e as variáveis sociodemográficas, não houve associação significativa para sexo $(p \leq 0,439)$; estado civil $(\mathrm{p} \leq$
$0,392)$; idade $(\mathrm{p} \leq 0,687)$; grau de parentesco $(\mathrm{p} \leq$ $0,540)$; escolaridade $(\mathrm{p} \leq 0,439)$; profissão $(\mathrm{p} \leq 0$, 392); moradia ( $\mathrm{p} \leq 0,221)$; número de filhos ( $\mathrm{p} \leq$ $0,223)$; com quem vive $(\mathrm{p} \leq 0,083)$ e nem com o estado de saúde atual $(\mathrm{p} \leq 0,269)$.

\section{CONCLUSÃO}

O estudo demonstrou que, em relação ao perfil e dados socioeconômicos dos cuidadores, a maioria é do sexo feminino, cônjuges e/ou filhos e com idade superior a 60 anos, dedicando em sua maioria 24 horas para o cuidado. Isto torna as condições de saúde destes ruins, apresentando um perfil de patologias como a artrite e o reumatismo, osteoporose, trombose, varizes e problemas gástricos, além da depressão, tornando necessário o uso de medicação diária.

A rotina de cuidados compreende a administração da própria vida do idoso demente. Em relação aos indicadores de depressão, transtornos mentais e sobrecarga do cuidado, demonstrou-se que o estado subjetivo de fadiga se manifesta através do cansaço, do esgotamento, da distração e da fraqueza, embora tenham relatado que se encontram despertos, estimulados e com energia suficiente, pois o próprio cuidado assim o exige. Uma minoria apresentou sobrecarga intensa.

Embora a maioria dos cuidadores tenha probabilidade de desenvolver transtornos psiquiátricos, os cuidadores mostraram alta disposição à resiliência, confirmando ser capazes de enfrentar as adversidades que a doença de Alzheimer traz. Também cabe destacar que houve associação significativa entre resiliência e idade, demonstrando que a resiliência se adquire com o passar da idade, pois a maioria dos cuidadores possui mais de 60 anos.

Acredita-se que a maior limitação deste estudo foi o reduzido número da amostra. Isto acontece porque, em geral, a família que tem idoso com mal de Alzheimer prefere deixá-lo no isolamento do seu lar ou institucionalizá-lo. Assim, recomendase a realização de um diagnóstico na cidade de Cruz Alta -RS, para identificar os casos de mal de Alzheimer e ampliar o estudo com cuidadores. 


\section{REFERÊNCIAS}

1. Instituto Brasileiro de Geografia e Estatística. 2010. Dados do Censo de 2010. Disponível em: <http://www.censo2010.ibge.gov.br/>. Acessado em 17 dez. 2010.

2. Lemos ND, Gazzola JM, Ramos LR. Cuidando do paciente com Alzheimer: o impacto da doença no cuidador. Saúde soc. 2006 set/dez; 15 (3): 170-179.

3. Duarte JE, Melo RV, Azevedo RS. Cuidando do cuidador. In: Moraes EN. Princípios Básicos de Geriatria e Gerontologia. Belo Horizonte:Coopmed; 2008.v.1,cap.26, p. 371-79.

4. Laranjeiras CASJ. Do vulnerável ser ao resiliente envelhecer: revisão de literatura. Psic.: Teor e Pesq $2007 \mathrm{jul} / \mathrm{set} ; 23$ (3): 327-32.

5. Gonçalves LHT, et al. Perfil da família cuidadora de idoso doente/fragilizado do contexto sociocultural de Florianópolis, SC. Texto contexto - enferm 2006; 15 (4): 570-77.

6. Grandjean E. Manual de ergonomia: adaptando o trabalho ao homem. 4. ed. Porto AlegreRS:Bookmann; 1998.

7. Mari J, Willians PA. A validity study of a psychiatric screening questionnaire (SRQ-20) in primary care in the city of São Paulo. Brit. J. Psychiatry 1986; 148: 23-6.

8. Scavufca M. Versão brasileira da Escala Burden Interview para avaliação de sobrecarga em cuidadores de indivíduos com doenças mentais. Rev Bras Psiquiatr 2002 mar;1 (24): 12-7.

9. Pesce RP, et al. Adaptação transcultural, confiabilidade e validade da escala de resiliência. Cad. Saúde Pública 2005 mar/abr; 21( 2); 436-38.

10. Neri AL. Cuidar de idosos no contexto da família: questões psicológicas e sociais. In: Neri AL (org.). As várias faces do cuidado e do bem estar do cuidador. São Paulo: Alínea; 2002. p. 9-63.

11. Haley WE. The family caregiver's role in Alzheimer's disease. Neurology 1997; 48 (5) Suppl 6; S25-S29.Disonível em: www.neurology.org/ content/48/5_Suppl_6/25S.abstract. Acessado em 17 dez.2010.

12. Garrido R, Menezes PR. Impacto em cuidadores de idosos com demência atendidos em um serviço psicogeriátrico. Revista de Saúde Pública 2004; 38 (6): 835-841. Disponível em: < http://www.scielo.br/ pdf/rsp/v38n6/12.pdf >. Acessado em 17 dez.2010.

13. Badia LX, et al. Calidad de vida, tiempo de dedicación y carga percibida por el cuidador principal informal del enfermo de Alzheimer. Rev Atenção Primaria à Saude 2004;34 (4); 170-177.
14. Silveira TM, Caldas CP, Carneiro TF. Caring for the highly dependent elderly in the community: a study on the main family caregivers. Cad Saude Publica 2006; 22 (8): 1629-38.

15. Roig MV, Abengozar MC, Serra E. La sobrecarga em los cuidadores principales de enfermos de Alzheimer. Anales de Psicología 1998 fev;14 (2); 215-27.

16. Andrieu S, et al. New assessment of dependency in demented patients: Impact on the quality of life in informal caregivers. Psychiatry Clin Neurosci. 2007 Jun; 61(3): 234-42.

17. Censis R. La mente rubata. Alzheimer's: i. costi e i bisogni assistenziali di una malattia familiare Milano: Franco Angeli; 1999. Disponível em: www. assistentisociali.org/.../alzheimer_bibliografia_ relativa.ph. Acesso em: 17 dez.2010.

18. Chaimowicz F. A saúde dos idosos brasileiros às vésperas do século XXI: problemas, projeções e alternativas. Rev Saúde Pública 2000 abr/jul; 31(2); 184-200, jul. 1997.

19. Camarano AA, et al. Famílias: espaço de compartilhamento de recursos e vulnerabilidades. In: Camarano AA. Os novos idosos brasileiros: muito além dos 60? Rio de Janeiro: IPEA; 2004.p. $137-167$

20. Baillie V, Norbeck JS, Barnes LE. Stress, social support and psychological distress of family caregivers of the elderly. Nursyng Research 1998;37(4); p. 217-22.

21. Karsch UM (org.). Envelhecimento com dependência: revelando cuidadores. São Paulo: EDUC; 1998.

22. Garrido R, Almeida OP. Distúrbios de comportamento em pacientes com demência: impacto sobre a vida do cuidador. Arq. NeuroPsiquiatr 1999;57 (2b); p. 427-43.

23. Karsch UM. Idosos Dependentes: famílias e cuidadores. Cad. Saúde Pública 2003 maio/jun.; 19(3):p.861-66.

24. Faleiros DAM. Cuidador de idosos com Doenças de Alzheimer: efeitos de grupos psico-educacionais e suporte domiciliar individualizado. 2009, 167 p. Dissertação de Mestrado. Pós-Graduação em Educação Especial do Centro de Educação e Ciências Humanas da Universidade federal de São Carlos. São Carlos-SP, 2009.

25. Freitas ICC, et al. Convivendo com o portador de Alzheimer: perspectivas do familiar cuidador. ReBEn 2008 jul/ago;61 (4); p. 508-13. 
26. Caldeira APS, RIBEIRO RCHM. O enfrentamento do cuidador do idoso com Alzheimer. Arq Ciênc Saúde 2004 abr/jun; 11(2); p. 1-6.

27. Cassis SVA, et al. Correlação entre o estresse do cuidador e as características clínicas do paciente portador de demência. RAMB 2007; 53 (6); p. 497-501.

28. Cruz MN, Hamdan AC. O impacto da doença de Alzheimer no cuidador. Psicol estud 2008 abr/jun; 13 (2); p. 223-29.

29. Melillo A, Ojeda ENS. (orgs.) Resiliência: descobrindo as próprias fortalezas. Tradução de Valério Campos. Porto Alegre: ARTMED; 2006.
30. Santos LMO, et al. resiliência e auto-estima em idosos assistidos na rede de atenção básica de saúde em Natal/RN. In: SEMANA DE HUMANIDADES, 7, Natal-RN, 01 a 05 de junho de 2009. Anais da XVII Semana de Humanidades. Natal-RN: UFRN, 2009. Disponível em:<http:// www.cchla.ufrn.br/humanidades2009/Anais/anais. html>. Acessado em 17 dez. 2010.

31. Garrido R, Almeida OP. Distúrbios de comportamento em pacientes com demência: impacto sobre a vida do cuidador. Arq NeuroPsiquiatr 1999; 57 (2b); p. 427-43.

Recebido: 01/3/2011

Revisado: 29/6/2011

Aprovado: 17/9/2011 